\title{
The investigation of protective concomitant immunity in murine Schistosomiasis mansoni ${ }^{*}$
}

\author{
Abdel-Moneim M. Salim ${ }^{1 \#, ~ A l a a ~ B . ~}$ Ismael $^{2,3}$ \\ ${ }^{1}$ Department of Medical Microbiology, Faculty of Applied Medical Sciences, Taif University, Turaba, KSA; \\ ${ }^{\#}$ Corresponding Author: salimabdelmoneim55@msn.com \\ ${ }^{2}$ Department of Medical Biotechnology, Faculty of Applied Medical Sciences, Taif University, Turaba, KSA \\ ${ }^{3}$ Department of Animal Medicine, Faculty of Veterinary Medicine, Zagazig University, Zagazig, Egypt
}

Received 5 March 2012; revised 5 April 2012; accepted 12 April 2012

\section{ABSTRACT}

The present study was conducted mainly to establish a new concomitant immunity model to Schistosoma mansoni in mice and assess its effects on the resistance of mice to a challenge infection with a possibility of using as a diagnostic marker. Three groups ( $A, B$ and $C$ ) of BALB/c mice were infected with a single dose of S. mansoni cercariae obtained from infected Biomphalaria glabrata snail. The group A mice were used as infected control group. The group $B$ mice were intraperitoneally injected with allylthiourea (ATU) 22 days post-primary infection then they were challenged 3 weeks post-ATU treatment. The group $C$ mice were challenged with the same number of cercariae 6 weeks post-primary infection. Perfusion of all mice was done 9 weeks after infection in order to obtain worm burdens. The livers of all mice were obtained for parasitological and pathological assessments. Our results showed that the group B mice had a $29.11 \%$ worm reduction rate, $25.37 \%$ liver egg reduction rate, and $37.48 \%$ granuloma size reduction rate compared to their respective controls. While the group C mice showed superior results and had a $54.66 \%$ worm reduction rate, $41.45 \%$ liver egg reduction rate, and $51.76 \%$ granuloma size reduction rate. It was concluded that these results described novel imaging methods that permit visualization of live schistosomes within their living hosts and may have important implications not only for epidemiological and diagnostic investigations, but also in designing control programs for schistosomiasis

\footnotetext{
*Funding: This study was supported by Faculty of Applied Medical Sciences, Taif University, Turaba, 21995, Saudi Arabia.

Conflict of interest: The authors declared no potential conflicts of interest with respect to the research, authorship, and/or publication of this article.
}

including anti-schistosome vaccine.

Keywords: Biomphalaria glabrata; Concomitant Immunity; Schistosoma mansoni; Mice; Protection

\section{INTRODUCTION}

Schistosomiasis (bilharziasis) has a significant impact on the health of both humans and their livestock, and the environments that they share. Human schistosomiasis, caused by infection with trematodes belonging to the genus fluke Schistosoma (blood flukes), is one of the most common tropical diseases. Despite the fact its global distribution has changed significantly, the disease is still a serious public health problem in many countries in which an estimated 779 million people are at risk for schistosomiasis, with 207 million infected in 76 countries and territories, and about 200,000 may die from the disease each year [1,2]. For animal schistosomiasis, it was evident that infected bovines and pigs with schistosomiasis (especially the younger animals) are the main infective sources. Results from experimental epidemiological studies showed that the density of infected snails in marshlands could drop to 0 or close to 0 through mass chemotherapy of domestic animals [3]. The observation and identification of forms of schistosomiasis passing between humans and animals is often a non-trivial matter (risk of emerging zoonosis), given the wide variety of definitive host species at risk of infection as well as morphological similarities between different schistosome species across several life stages [4]. In terms of control strategies for schistosomiasis, enormous efforts have been made to develop effective anti-schistosome vaccines, but none is available at the present time. This may be due to the complexity of the schistosome and its life cycle, in addition to the pathology and morbidity in this chronic disease result from the host immune inflammatory response to parasite eggs trapped in the liver and other organs $[5,6]$. A reduction in worm numbers is the 
"gold standard" for anti-schistosome vaccine development. Concomitant immunity $(\mathrm{CI})$ has received an attention in schistosomiasis and thus we shall motivate this paper with reference to this disease.

CI is characterized by the ability of a host to mount an effective defense against larval stages whilst being unable to clear a persistent burden of adult worms [7]. From the parasite perspective, the generation of CI may be beneficial as it reduces interspecies competition within the host. Even if it is beneficial to established parasites, CI may not be a parasite adaptation. It may, alternatively, be an incidental effect of an immunological reaction that is shaped by distinct host and parasite adaptations. Smithers and Terry (1969) transferred adult schistosomes (Schistosoma mansoni) from infected to uninfected monkeys in a series of surgical transplant experiments and later challenged the recipients with large numbers of cercariae. The recipient monkeys were almost totally resistant to the challenge infection, despite having no previous experience of larval schistosome stages, yet were unable to reject the implanted adults. Thus, a curious situation is exposed wherein adult worms are exempt from the immune response that they themselves provoke [8].

Schistosomal antigens are secreted and excreted into the blood from the established adult worms and from the migrating eggs, thereby providing a potent stimulus for the immune system. This response was found against challenge infection in rhesus monkeys, hamsters [7], rabbits [9] and mice [10]. Larval exposure appears to be central to the CI found in lymphatic filariasis [11]. Thus, there are two distinct sources of antigen that are acting to strengthen immunity against larval challenge and form the mechanistic basis of CI: larval-triggered and adulttriggered CI. From an evolutionary perspective, the latter is more unknowable.

Adult-triggered CI may be interpreted as similar to vaccination; relatively long-lived adult worms vaccinate their host against subsequent challenge infection. Autopsy data on Schistosoma haematobium infection in Egypt are suggestive of an even stronger impact of parasite density on individual fecundity. Here the mean egg output of heavily infected host appeared to be lower than that of host with fewer worms, this was assumed to result from acquired immune responses to both past and current infection, [12]. These studies thereby provided a strong immunological basis for CI. However, a few authors have suggested that the mechanism of resistance may be related to the hepatic pathology caused by the primary infection $[13,14]$. The vascular pathology might enable the challenge schistosomula to escape from the hepatic portal system, thus preventing normal parasite sequestration and maturation in the liver.

The overall objective of this study was to test the ef- fect of previous infection with $S$. mansoni on the resistance of mice to a challenge infection (CI) by comparing the following parameters with that obtained from agematched control mice: 1) Parasitological parameters, such as the numbers of the recovered adult worms and the numbers of eggs retained in the liver in term of egg per gram (EPG) liver tissue. 2) Pathological parameters such as granuloma size.

On the other hand, phenol oxidase has been implicated in the process of eggshell formation $[15,16]$. We hypothesized that allylthiourea (ATU), the most effective phenol oxidase inhibitor, may block the formation of $S$. mansoni eggshell. Here we report the establishment of a novel model based on ATU abbreviated infection that can be used to explore the real mechanism of immunity to reinfection with $S$. mansoni.

\section{MATERIALS AND METHODS}

\subsection{Animals}

$\mathrm{BALB} / \mathrm{c}$ male, naïve mice aged six to seven weeks were used in all experiments. The animals were divided randomly into 3 groups of 10 animals each, housed in polypropylene cages $(445 \times 280 \times 125 \mathrm{~mm}$; Labotec, Johannesburg, South Africa) on wood shavings, 10 mice per cage. Each housed room is approximately $15 \mathrm{M}^{2}$ in area and is maintained under positive pressure, having an average temperature of $25^{\circ} \mathrm{C} \pm 2^{\circ} \mathrm{C}$ with $12 \mathrm{~h} / 12 \mathrm{~h}$ light/ dark cycle, humidity of 50 - 600/0 and receiving $10-13$ air changes per hour. Tap water and standard rodent food pellets (Epol, Pietermaritzburg, South Africa) were provided "ad libitum".

\subsection{Parasites and Snails Materials}

The $S$. mansoni cercariae were obtained from laboratory raised and infected Biomphalaria glabrata snail as previously described [17] with modifications. Briefly, cercaria shedding was induced by subjecting infected, water-immersed snails to light for 1.5 hours. The cercariae were concentrated by cooling and low speed centrifugation. After collecting from the infected snails, the cercariae were placed on cover slip and the number of cercariae used for each animal was accurately counted under a dissecting microscope.

\subsection{Infection of Mice}

Each animal was exposed to percutaneous infection by approximately $100 \pm 2 S$. mansoni cercariae using the paddling method [18]. Briefly, animals were placed individually into $600 \mathrm{ml}$ plastic beakers containing $80 \mathrm{ml}$ of dechlorinated tap water and the appropriate number of cercariae. After $45 \mathrm{~min}$, they were returned to their cages. All animals were infected on the same day within a pe- 
riod of 5 hours. Remaining cercariae were counted and mice receiving less than $95 \%$ of the cercariae were excluded from the experiment [19]. The time between the primary and the challenge infection was six weeks, except where stated otherwise.

\subsection{Experimental Design}

After cercarial exposure, the animals were randomly divided into 3 groups, each with 10 mice, as follows:

1) Group A (Infected control-mice): these are mice with primary infection. They were infected and given distilled water, day after day, from the $1^{\text {st }}$ day of infection till the end of the $9^{\text {th }}$ week post-infection.

2) Group B (Allylthiourea, ATU, treated mice): Twentytwo days post-primary infection, mice were intraperitoneally injected with ATU at a dose of $200 \mathrm{mg} / \mathrm{kg}$ every other day. The mice were challenged with $S$. mansoni cercariae 3 weeks post-ATU treatment.

3) Group C (Concomitant mice): was designed to evaluate the effect of the concomitant immunity to challenge infection. Mice in this group were challenged with the same number of cercariae six weeks after primary infection.

\subsection{Adult Worm Recovery}

Nine weeks after cercarial exposure, all mice were sacrificed by cervical dislocation, and worms were recovered from the portal system and mesenteric veins by vascular perfusion technique [20]. The perfused saline plus blood drained through the portal vein was recovered in a beaker, and left to sediment. The supernatant was discharged and the sediment washed out twice with saline. After washings the recovered worms were counted under a magnifying lens. The percentage of reduction $(\%$ protection) for each group and each experiment was calculated according to the following formula: \% reduction $(\%$ protection $)=($ mean worm count in control group mean worm count in concomitant or ATU group) $\times 100 /$ (mean worm count in control group).

\subsection{Liver Egg Counts}

The egg count of $S$. mansoni in the perfused liver of mice was estimated after alkali digestion as previously described [21] with modifications. Briefly, at the time of perfusion, whole livers of experimental and control mice were weighed and a known portion $(0.5 \mathrm{~g})$ was removed to a screw cap glass tube and frozen until digestion. The frozen sample of the liver was minced. For digestion 5 $\mathrm{ml}$ of $5 \% \mathrm{KOH}$ (Potassium hydroxide) was added to each tube and incubated at $37^{\circ} \mathrm{C}$ until the tissue was completely digested (10 - 12 hours). Egg counts of three $1 \mathrm{ml}$ portion of the suspension were determined by micro- scopic examination at $40 \times$. Total egg counts were expressed for each group of mice as the mean number of eggs/gram of mouse liver (the EPG liver).

\subsection{Liver Granuloma Measurement}

Granuloma diameters were measures in histological sections [22]. After portal perfusion, part of the liver tissue was immediately fixed in $10 \%$ buffered formalin solution and processed in paraffin blocks. Paraffin sections were routinely stained with hematoxylin-eosin (HE) for microscopic examination. In each histological section, 10 granulomas with visible central eggs were randomly selected; their diameters were measured at $10 \times$ magnification using calibrated ocular micrometer. Two perpendicular maximal diameters were measured, getting the mean diameter for each granuloma and then calculating the mean granuloma diameter for each mouse in a group and the sizes in a group were averaged for statistical comparison.

\subsection{Statistical Analysis}

Data were presented as mean \pm SD. Group means and standard deviations for all groups were calculated and compared. Statistical significance was assessed by Student's t-test. Values of $P<0.05$ were taken to be significant.

\section{RESULTS}

In order to measure the degree of protection conferred by concomitant immunity, Group $\mathrm{C}$ mice were challenged with $S$. mansoni cercariae. Worm burden, egg count in the liver, and hepatic granuloma size were determined for each group. The results are shown in Tables $\mathbf{1}, \mathbf{2}$ and $\mathbf{3}$ and Figure $\mathbf{1 .}$

\subsection{Worm Recovery}

Infections were obtained in all mice. Immature worms were recovered from mice in all groups. These immature worms were included in figures for total worm burdens. Statistical analysis of the obtained data, presented in Table 1 , showed that the application of concomitant immunity evoked a highly significant reduction $(54.66 \% ; P<$ $0.001)$ in the mean worm count nine weeks after cercarial exposure as compared to the infected control-mice. While ATU-treated mice showed a low significant reduction $(29.11 \% ; P<0.05)$ as compared to the infected control-mice

\subsection{Liver Egg Counts}

The concomitant mice (Group C) showed a highly significant decrease $(41.45 \%, P<0.001)$ in the total liver 
Table 1. Comparison of worm numbers recovered from different groups.

\begin{tabular}{ccccc}
\hline Group & Infection status & Worm burden & \% Protection & $P$-value \\
\hline A & Infected control-mice & $60.66 \pm 5.85$ & - & - \\
B & ATU-treated mice & $43.00 \pm 4.24$ & 29.11 & $<0.05$ \\
C & Concomitant mice & $27.50 \pm 5.00$ & 54.66 & $<0.001$ \\
\hline
\end{tabular}

The number of cercariae used for primary/challenge infections was 100; Results from one of three similar experiments are shown.

Table 2. Mean number of tissue egg counts and the percentage reduction in challenge egg deposition in the liver.

\begin{tabular}{ccccc}
\hline Group & Infection status & Mean EPG & \% Reduction & $P$-value \\
\hline A & Infected control-mice & $800.66 \pm 19.00$ & - & - \\
B & ATU-treated mice & $597.50 \pm 38.89$ & 25.37 & $<0.01$ \\
C & Concomitant mice & $468.75 \pm 42.69$ & 41.45 & $<0.001$ \\
\hline
\end{tabular}

Results from one of three similar experiments are shown.

Table 3. Granuloma sizes in different groups of mice 9 weeks after a single dose of 100 cercariae/mice.

\begin{tabular}{ccccc}
\hline Group & Infection status & Granuloma size $(\mu \mathrm{m})$ & \% Reduction & $P$-value \\
\hline A & Infected control-mice & $102.2 \pm 17.14$ & - & - \\
B & ATU-treated mice & $63.90 \pm 4.10$ & 37.48 & $<0.05$ \\
C & Concomitant mice & $49.30 \pm 6.68$ & 51.76 & $<0.01$ \\
\hline
\end{tabular}

Results from one of three similar experiments are shown.

eggs as compared to the infected control-mice (Table 2). While ATU-treated mice showed a low significant reduction $(25.37 \% ; P<0.01)$ as compared to the infected control-mice.

\subsection{Granuloma Size}

In spite of the presence of lesions throughout the liver of all mice, however, significant differences in the tissue response to schistosome eggs were noted (Figure 1 and Table 3). The concomitant mice developed lesions that were, on average, $51.76 \%$ smaller than those formed in infected control-mice $(t=5.765, P<0.01)$. While ATUtreated mice showed moderate significant reduction in granuloma size $(37.48 \% ; P<0.05)$ when compared to the infected control-mice.

\section{DISCUSSION}

Infection with Schistosoma spp. in the rat has been extensively studied, and offers several biological and immunological features which render this host an ideal model for the study of this parasite. This study confirms the possibility of the concomitant immunity (CI) as one of these features. Previous experimental studies on CI indicate that this feature is an immunological process and is called "self-cure phenomenon" [23,24]. It was observed between the $4^{\text {th }}$ and the $11^{\text {th }}$ week as measured by the liver perfusion technique $[23,25,26]$, or by the lung recovery method [27]. The exact mechanism of CI as a phase of acquired immunity is still unclear, but many in vitro studies suggested the involvement of antibodies and non-sensitized cells in this process [28-30].

This study indicates that we have successfully established a new concomitant immunity model in mice with the following characteristics: resistance in previously infected mice to a challenge infection led to a $54.66 \%$ worm reduction rate, $41.45 \%$ liver egg reduction rate, and $51.76 \%$ granuloma size reduction rate, as compared to naive mice facing a similar challenge infection. Therefore, mice with previous $S$. mansoni infection are able to mount a very strong regulatory response to a challenge infection, which could be termed concomitant immunity.

The development of CI found in mice could be due to the following reasons: first, the inhibition of egg production eliminate the excretion of soluble egg antigens (SEA) and, hence, the production of blocking antibodies as shown previously in NM mice [31] which may explain the enhancement of CI. Second, comparing with the irradiated vaccine model, established by McLaren and Rogers, the protection against reinfection due to concomitant immunity is dependent on the living adult worms in NM mice [32]. This is supported by [33] who concluded that adult worms might promote anti-larval 

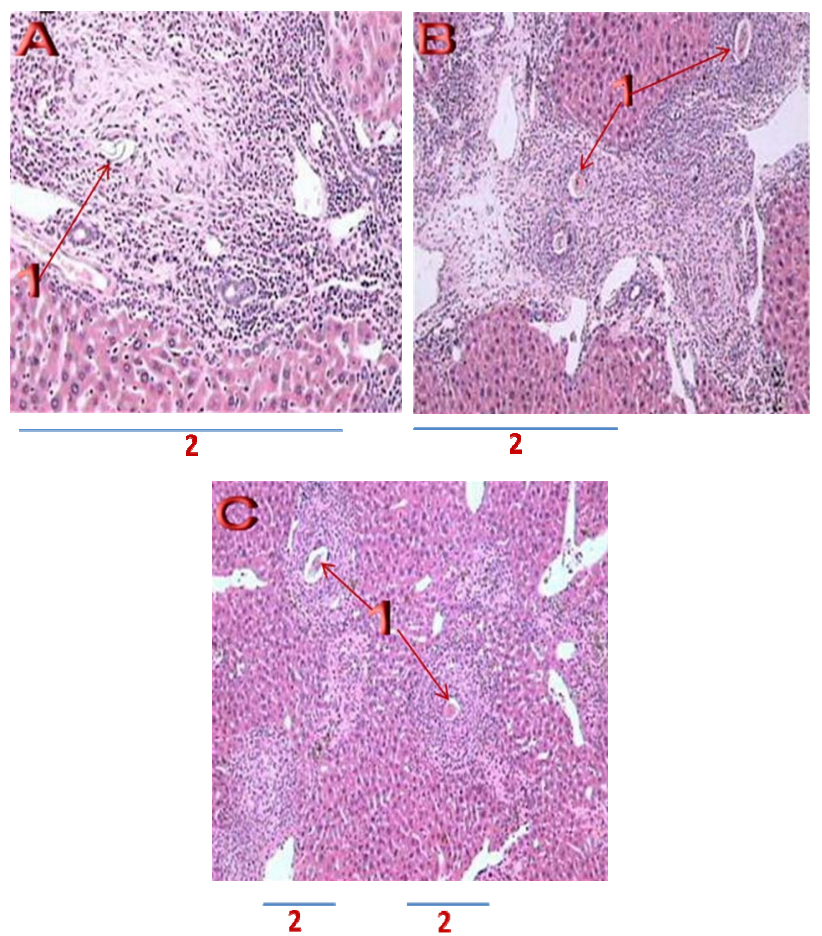

Figure 1. Photomicrographs of hepatic granulomas of 9 weeks infected control-mice (A), ATU-treated mice (B) and concomitant mice (C). Liver sections stained with hematoxylin and eosin $(100 \times)$ showed granuloma size and liver necrosis. 1: Schistosome eggs, 2: Granuloma size.

immunity via the release of antigens, thus creating a barrier against continuous infection and limiting burden size within the host. This adult-triggered CI may be interpreted as similar to vaccination; relatively long-lived adult worms "vaccinate" their host against subsequent challenge infection.

The present study demonstrated that the CI decreases tissue egg load by $41.45 \%$. The reducing eggs load in the tissues can significantly relieve the syndrome of schistosomiasis including inflammation, granuloma, and tissue fibrosis [34]. Moreover the concomitant mice showed superior results when compared to ATU-treated mice especially in percentage of egg reduction $(41.45 \%$ vs. $25.37 \%$ ), although ATU is the most effective phenol oxidase inhibitor that may block the formation of $S$. mansoni eggshell [16].

From the above discussion it seems that, our results strongly support the hypothesis first proposed by Smithers and Terry in 1969 that the resistance of the definitive host to schistosome reinfection is related to concomitant immunity induced by adult worms in vivo, which not affect the established adult worms but does affect reinfection with the parasite in the larval stage [7].

It was concluded that these results described novel imaging methods that permit visualization of live schistosomes within their living hosts and may help to eluci- date mechanisms of infection also be of value not only for epidemiological and diagnostic investigations, but also in designing government control programs for schistosomiasis.

\section{REFERENCES}

[1] Steinmann, P., Keiser, J., Bos, R., et al. (2006) Schistosomiasis and water resources development, systematic review, meta-analysis, and estimates of people at risk. Lancet Infectious Diseases, 6, 411-425. doi:10.1016/S1473-3099(06)70521-7

[2] World Health Organization (2008) The social context of schistosomiasis and its control: An introduction and annotated bibliography.

[3] Zhou, X., Chen, M., Don, Mc. and Robert, B. (2002) Schistosomiasis control in the 21st century: Proceedings of the International Symposium on Schistosomiasis, Shanghai, 4-6 July 2001. Acta Tropica, 82, 95-114.

[4] Claire, J.S., Andrew, P.D. and Stothard, J.R. (2012) Out of animals and back again: Schistosomiasis as a zoonosis in Africa. In: Rokni, M.B., Ed., Schistosomiasis, 1st Edition, InTech Publisher, Janeza Trdine, Rijeka, Croatia. 209-230.

[5] Bergquist, N.R. (2002) Schistosomiasis: From risk assessment to control. Trends in Parasitology, 18, 309-314. doi:10.1016/S1471-4922(02)02301-2

[6] Capron, A., Riveau, G.J., Bartley, P.B. and McManus, D.P. (2002) Prospects for a schistosome vaccine. Current Drug Targets: Immune, Endocrine \& Metabolic Disorders, 2, 281-290.

[7] Smithers, S.R. and Terry, R.J. (1969) Immunity in Schistosomiasis. Annals of the New York Academy of Sciences, 160, 826-840. doi:10.1111/j.1749-6632.1969.tb15904.x

[8] Smithers, S.R. and Terry, R.J. (1965) The infection of laboratory hosts with cercariae of Schistosoma mansoni and the recovery of the adult worms. Parasitology, 55, 695-700.

[9] Tendler, M., Pinto, R.M., Lima, A.O., et al. (1986) Schistosoma mansoni-vaccination with adult worm antigens. International Journal for Parasitology, 16, 347-352. doi:10.1016/0020-7519(86)90113-X

[10] Cutts, I. and Wilson, R.A. (1997) The protein antigens secreted in vivo by adult male Schistosoma mansoni. Parasitology, 14, 245-255. doi:10.1017/S0031182096008438

[11] Maizels, R.M. and Lawrence, R.A. (1991) Immunological tolerance-The key feature in human filariasis. Parasitology Today, 7, 271-276. doi:10.1016/0169-4758(91)90093-4

[12] Cheever, A.W., Kamel, I.A., Elwi, A.M., et al. (1978) Schistosoma mansoni and Schistosoma haematobium in Egypt. American Journal of Tropical Medicine and Hygiene, 27, 55-75.

[13] Wilson, R.A., Coulson, P.S. and McHugh, S.M. (1983) A significant part of the "concomitant immunity" of mice to Schistosoma mansoni is the consequence of a leaky he- 
patic portal system, not immune killing. Parasite Immunology, 5, 595-601. doi:10.1111/j.1365-3024.1983.tb00776.x

[14] McHugh, S.M., Coulson, P.S. and Wilson, R.A.P. (1983) Athologically induced alterations in the dimensions of the hepatic portal vasculature of mice infected with Schistosoma mansoni. Parasit X, 94, 69-80.

[15] Seed, J.L. and Bennett, J.L. (1980) Schistosoma mansoni: Phenol oxidase's role in eggshell formation. Experimental Parasitology, 49, 430-441. doi:10.1016/0014-4894(80)90077-6

[16] Eshete, F. and LoVerde, P.T. (1993) Characteristics of phenol oxidase of Schistosoma mansoni and its functional implications in eggshell synthesis. Journal of Parasitology, 79, 309-317. doi:10.2307/3283563

[17] Schubert, M. (1948) Conditions for drug testing in experimental Schistosomiasis mansoni in mice. American Journal of Tropical Medicine, 28, 121-136.

[18] Frandsen, F. (1981) Cultivation of schistosomes for chemotherapeutic studies. Acta Pharmacologica et Toxicologica, 49, 118-122. doi:10.1111/j.1600-0773.1981.tb03262.x

[19] Allam, G. (2009) Immunomodulatory effects of curcum in treatment on murine Schistosomiasis mansoni. Immunobiology, 214, 712-727. doi:10.1016/j.imbio.2008.11.017

[20] Duvall, R.H. and DeWitt, W.B. (1967) An improved perfusion technique for recovering adult schistosomes from laboratory animals. American Journal of Tropical Medicine and Hygiene, 16, 483-486.

[21] Cheever, A.W. (1968) Condition affecting the accuracy of potassium hydroxide digestion techniques for counting Schistosoma mansoni eggs in tissues. Bulletin of World Health Organization, 39, 328-331.

[22] Ohmae, H., Tanaka, M., Nara, T, et al. (1991) Serologic and ultrasonographic parameters of praziquantel treatment of hepatic fibrosis in Schistosoma japonicum infection. American Journal of Tropical Medicine and Hygiene, 45, 350-359.

[23] Phillips, S.M., Reid, W.A., Bruce, J.I., et al. (1975) The cellular and humoral immune response to $S$. mansoni infections in inbred rats. I. Mechanisms during initial exposure. Cellular Immunology, 19, 99-103. doi:10.1016/0008-8749(75)90295-6

[24] Cioli, D. and Dennert, G. (1976) The course of S. mansoni infection in thymectomized rats. Immunology, 117, 59-63.

[25] Smithers, S.R. and Terry, R.J. (1965) Acquired resistance to experimental infections of $S$. mansoni in the albino rat. Parasitology, 55, 711-719.

[26] Knopf, P.M., Nutman, T.B. and Reasoner, J.A. (1977) $S$. mansoni: Resistance to reinfection in the rat. Experimental Parasitology, 41, 74-81. doi:10.1016/0014-4894(77)90131-X

[27] Perez, H.A., Clegg, J.A. and Smithers, S.R. (1974) Acquired immunity to Schistosoma mansoni in the rat: Measurement of immunity by the lung recovery technique. Parasitology, 69, 34915-34920. doi:10.1017/S0031182000063046

[28] Dean, D.A., Wistar, R. and MuRuell, K.D. (1974) Combined in vitro effects of rat antibody and neutrophilic leukocytes on schistosomula of $S$. mansoni. American Journal of Tropical Medicine and Hygiene, 23, 420-427.

[29] Perez, H.A. and Smithers, S.R. (1977) Schistosoma mansoni in the rat: The adherence of macrophages to schistosomula in vitro after sensitization with immune serum. International Journal of Parasitology, 7, 315-318. doi:10.1016/0020-7519(77)90040-6

[30] Capron, A., Dessaint, J.P., Capron, M. and Bazin, H. (1975) Specific IgE antibodies in immune adherence of normal macrophages to Schistosoma mansoni schistosomules. Nature, 253, 47-52. doi: $10.1038 / 253474 \mathrm{a} 0$

[31] Yi, X.Y., Simpson, A.J., de Rossi, R. and Smithers, S.R. (1986) The presence of antibody in mice chronically infected with Schistosoma mansoni which blocks in vitro killing of schistosomula. Journal of Immunology, 137, 3955-3958.

[32] McLaren, D.J. and Rogers, M.V. (1986) Schistosoma mansoni: Liver phase challenge attrition is a stage-dependent phenomenon in guinea-pigs vaccinated with highly irradiated cercariae. Parasite Immunology, 8, 307-318. doi:10.1111/j.1365-3024.1986.tb00848.x

[33] Brown, S.P. and Grenfell, B.T. (2001) An unlikely partnership: Parasites, concomitant immunity and host defense. Proceedings of the Royal Society B: Biological Sciences, 268, 2543-2549. doi:10.1098/rspb.2001.1821

[34] Huang, H.Q., Li, S.C., Qin, Z.H., et al. (2005) Synthesis and bioactivities of two multiple antigen peptides as potential vaccine against Schistosoma. Bioorganic \& Medicinal Chemistry Letters, 15, 2415-2419. doi:10.1016/j.bmcl.2005.01.040

[35] Phillips, S.M., Reid, W.A. and Sadun, E.H. (1977) The cellular and humoral immune response to $S$. mansoni infection in inbred rats. II. Mechanisms during re-exposure. Cellular Immunology, 28, 75-83. 